\title{
Étude du graphe divisoriel 4
}

\author{
Pierre Mazet et Eric Saias
}

\section{Introduction}

Appelons chaîne-permutation du graphe divisoriel de $\mathbb{N}^{*}$ (ou plus simplement chaîne-permutation) toute application bijective $f: \mathbb{N}^{*} \rightarrow \mathbb{N}^{*}$ telle que $f(n)$ est un diviseur ou un multiple de $f(n+1)$ pour tout entier $n \geq 1$. Il est facile de construire des chaînes-permutations. Voici les premières valeurs de l'une d'entre elles :

$1-2-(2 \times 3)-3-(3 \times 4)-4-(4 \times 5)-5-(5 \times 7)-7-(7 \times 8)-8 \cdots$

On a ici $\lim \sup _{n \rightarrow+\infty} f(n) / n^{2}=1 / 4$. Le résultat ci-dessous montre que la convergence vers l'infini de $f(n)$ peut être beaucoup plus lente.

\section{Théorème.}

Il existe une constante $c_{1}$ et une chaîne-permutation $f$ telles que pour tout $n \geq 2$, on a

$$
f(n) \leq c_{1} n(\log n)^{2}
$$

Intéressons-nous maintenant à des permutations de $\mathbb{N}^{*}$ qui ne sont pas nécessairement des chaînes-permutations. Notons $[a, b]$ le plus petit commun multiple des entiers $a$ et $b$. En 1983, Erdös, Freud et Hegyvari ont montré qu'il existe une constante $c_{2}$ et une permutation $f$ de $\mathbb{N}^{*}$ telles que pour tout $n \geq 3$, on a

$$
[f(n), f(n+1)] \leq n \exp \left\{c_{2} \sqrt{\log n} \log \log n\right\}
$$

(voir le théorème 3 de [2]). En modifiant légèrement la permutation choisie par Erdös, Freud et Hegyvari, Chen et Ji [1] ont montré en 2011 que l'on peut remplacer la fonction à l'intérieur de l'accolade par $(2 \sqrt{2}+o(1)) \sqrt{\log n \log \log n}$. 
Il découle immédiatement de notre théorème une amélioration de leur résultat.

\section{Corollaire.}

Il existe une constante $c_{3}$ et une permutation $f$ de $\mathbb{N}^{*}$ telles que pour tout $n \geq 2$, on a

$$
[f(n), f(n+1)] \leq c_{3} n(\log n)^{2} .
$$

Signalons que Tenenbaum [6] conjecture que l'on peut remplacer l'expression à droite du signe $\leq$ dans cette dernière formule par $n(\log n)^{1+o(1)}$. L'exposant 1 de $\log n$ serait alors optimal car on sait que (théorème 3 de [4]) pour toute permutation $f$ de $\mathbb{N}^{*}$, on a

$$
\limsup _{n \rightarrow+\infty} \frac{[f(n), f(n+1)]}{n \log n}>0 .
$$

Pour établir le théorème, on utilise une construction de chaîne finie due à Tenenbaum (voir le paragraphe 4 de [6]) et un résultat relatif aux entiers à diviseurs denses (théorème 1 de [3]) (pour la définition de ces termes, voir le paragraphe suivant). Même si cela ne nous est pas utile ici, signalons au passage que le récent travail de Weingartner [7 fournit un équivalent asymptotique de la fonction de comptage des entiers à diviseurs $y$-denses, uniforme en $y$.

\section{Notations}

Appelons chaîne toute application injective $f: \mathbb{N}^{*} \rightarrow \mathbb{N}^{*}$ telle que $f(n)$ est un diviseur ou un multiple de $f(n+1)$ pour tout entier $n \geq 1$. Appelons aussi chaîne finie de longueur $l$ tout $l$-uplet $\mathcal{C}=a_{1}, a_{2}, \cdots, a_{l}$ d'entiers positifs deux à deux distincts et tels que $a_{i}$ est un diviseur ou un multiple de $a_{i+1}$ pour tout entier $i$ de l'intervalle $[1, l-1]$. On notera longueur $(\mathcal{C}):=l$.

On note pour tout entier $n \geq 2, P^{+}(n)$ (respectivement $P^{-}(n)$ ) le plus grand (resp. petit) facteur premier de n. On pose de plus $P^{+}(1)=1$.

On note 


$$
F(n):=\left\{\begin{array}{cc}
1 & (n=1) \\
\max \left\{d P^{-}(d): d \mid n, d>1\right\} & (n \geq 2) .
\end{array}\right.
$$

On dit qu'un entier est à diviseurs $y$-denses si $F(n) \leq y n$. Cette dénomination provient de l'identité

$$
\frac{F(n)}{n}=\max _{1 \leq i<\tau(n)} \frac{d_{i+1}(n)}{d_{i}(n)}
$$

où $1=d_{1}(n)<d_{2}(n)<\cdots<d_{\tau(n)}(n)=n$ désigne la suite croissante des diviseurs de $n$ (voir le lemme 2.2 de [5]).

\section{Preuve du théorème}

Soit $p$ un nombre premier.

Au paragraphe 4 de [6], Tenenbaum construit une famille de chaînes finies $\Gamma(x, p)$ pour tout réel $x \geq 2$. On s'intéresse ici au cas particulier $x=2 p^{2}$ pour lequel on a les quatre propriétés suivantes.

$\Gamma(8,2)=1-2$.

Pour tout $p \geq 3, \Gamma\left(2 p^{2}, p\right)=1-p-\cdots-2$.

Pour tout $p, \Gamma\left(2 p^{2}, p\right)$ est constitué d'entiers $m$ sans facteur carré et tels que $m \leq 2 p^{2}, P^{+}(m) \leq p$.

Pour tout $p, \Gamma\left(2 p^{2}, p\right)$ contient tous les entiers $m$ sans facteur carré et tels que $F(m) \leq p^{2}$.

Cette dernière propriété combinée avec le théorème 1 de [3] entraîne l'existence d'une constante $c>0$ telle que pour tout $p$

$$
\text { longueur }\left(\Gamma\left(2 p^{2}, p\right)\right) \geq c p^{2} / \log p \text {. }
$$

Nous modifions légèrement $\Gamma\left(2 p^{2}, p\right)$ en considérant la chaîne finie $\mathcal{D}(p)$ obtenue en déplaçant dans $\Gamma\left(2 p^{2}, p\right)$ le 1 initial pour le mettre en final, puis en multipliant le tout par le nombre premier $p^{*}$ suivant immédiatement $p$. On a donc pour $p \geq 3, \mathcal{D}(p)=p^{*} p-\cdots-2 p^{*}-p^{*}$.

Soit $f_{0}$ la suite commençant à 1 , puis obtenue par concaténation des $\mathcal{D}(p)$.

$$
f_{0}: 1-\mathcal{D}(2)-\mathcal{D}(3)-\mathcal{D}(5)-\mathcal{D}(7)-\cdots
$$

On vérifie que $f_{0}$ est une chaîne formée d'entiers sans facteur carré. On pose $q_{0}=1$. 
On construit alors par récurrence une suite croissante $\left(q_{k}\right)_{k \geq 0}$ de nombres égaux à 1 ou premiers et des chaînes $f_{k}$ construites à partir de $f_{0}$ en remplaçant, pour $p \leq q_{k}$, la chaîne finie $\mathcal{D}(p)$ par une chaîne finie $\mathcal{C}(p)$ à définir.

Supposons construits $q_{0} \leq q_{1} \leq \cdots \leq q_{k-1}, \mathcal{C}(p)$ pour $p \leq q_{k-1}$ ainsi donc que $f_{0}, f_{1}, \cdots, f_{k-1}$. Si $k$ est dans l'image de $f_{k-1}$, on pose $q_{k}=q_{k-1}$, il n'y a pas de nouvelle chaîne finie $\mathcal{C}(p)$ à définir et donc $f_{k}=f_{k-1}$.

On suppose dorénavant que $k$ n'est pas dans l'image de $f_{k-1}$.

On choisit $q_{k}$ un nombre premier tel que $q_{k}>q_{k-1}$ et

$$
q_{k} \geq k^{2}
$$

On définit alors $\mathcal{C}(p)$ pour $q_{k-1}<p \leq q_{k}$ de la manière suivante.

Si $q_{k-1}<p<q_{k}$, on pose $\mathcal{C}(p)=\mathcal{D}(p)$.

Si $p=q_{k}$, on choisit

$$
\mathcal{C}(p)=p k^{2}-k-p^{*} p k^{2}-\mathcal{D}(p)
$$

Le fait que $\mathcal{D}(p)$ est formée d'entiers sans facteur carré assure que les éléments de $\mathcal{C}(p)$ sont deux à deux distincts et donc que $\mathcal{C}(p)$ est une chaîne finie.

On pose alors

$$
f_{k}: 1-\mathcal{C}(2)-\mathcal{C}(3)-\cdots-\mathcal{C}\left(q_{k}\right)-\mathcal{D}\left(q_{k}^{*}\right)-\mathcal{D}\left(q_{k}^{* *}\right)-\cdots
$$

et on vérifie que c'est une chaîne. On définit enfin

$$
f=\lim _{k \rightarrow+\infty} f_{k}=1-\mathcal{C}(2)-\mathcal{C}(3)-\mathcal{C}(5)-\cdots-\mathcal{C}(p)-\cdots
$$

et on vérifie que c'est une chaîne-permutation.

Le début de cette chaîne-permutation est formée des trois entiers $1-6-3$, et est suivie de la chaîne finie $\mathcal{C}(3)$. Soit $n \geq 4$; il existe donc un nombre premier $p$ tel que $f(n) \in \mathcal{C}\left(p^{*}\right)$. En utilisant notamment (3.2), on a $f(n) \leq 2 p^{* 2} p^{* *} \asymp p^{3}$. Par ailleurs notons $r$ un nombre premier générique. On a en utilisant (3.1) et le théorème des nombres premiers

$$
n \geq \sum_{r \leq p} \text { longueur }(\mathcal{C}(r)) \gg \sum_{r \leq p} r^{2} / \log r \asymp p^{3} /(\log p)^{2} .
$$


On en déduit que $f(n) \ll n(\log n)^{2}$. Cela conclut la démonstration du théorème.

\section{Références}

[1] Y.-G. CHEn and C.-S. JI. - The permutation of integers with small least common multiple of two subsequent terms, Acta Math. Hungarica 132 (2011), 307-309.

[2] P. ERdös, R. Freud and N.Hegyvari. - Arithmetical properties of permutation of integers, Acta Math. Hungarica 41 (1983), 169-176.

[3] E. SAIAS.- Entiers à diviseurs denses 1, J. Number Theory 62 (1997), 163-191.

[4] E. SAIAS.- Applications des entiers à diviseurs denses, Acta Arithmetica 83 (1998), 225-240.

[5] G. Tenenbaum.- Sur un problème de crible et ses applications, Ann. Sci. Ecole Norm. Sup. (4) 19 (1986), 1-30.

[6] G. Tenenbaum.- Sur un problème de crible et ses applications, 2. Corrigendum et étude du graphe divisoriel, Ann. Sci. Ecole Norm. Sup. (4) 28 (1995), 115-127.

[7] A. Weingartner.- Practical numbers and the distribution of divisors, Quart. J. Maths 66 (2015), 743-758.

Pierre Mazet $\quad$ Eric Saias

Laboratoire de Probabilités, Statistique et Modélisation, Sorbonne Université, 4, place Jussieu, 75252 Paris Cedex 05 (France)

piermazet@laposte.net eric.saias@upmc.fr 